\title{
Le Nécrophile de Gabrielle Wittkop : la perversion, l'abjection et l'antirelationalité queer
}

\section{Gabriel RÉMY-HANDFIELD, Université de Montréal}

Gabrielle Wittkop (1920-2002) est une romancière française peu connue du grand public et sa réception dans le milieu académique se fait aussi de manière discrète ${ }^{1}$. Se revendiquant du marquis de Sade, elle produit une œuvre littéraire dérangeante et obscène dans laquelle on retrace une obsession constante pour la mort. Cette obsession traverse la majorité de sa création artistique, que ce soit avec La marchande d'enfants (2005) ou La mort de C (2001). Dans les romans de Wittkop, la sexualité est souvent envisagée comme un acte transgressif qui vient déjouer les lois morales et sociales. Selon nous, son écriture cherche à contester et surtout à transgresser les normes en matière de sexualité, qui sont imposées par la société. Son premier roman, Le Nécrophile (1972), raconte le quotidien d'un antiquaire dénommé Lucien N qui se réclame d'une pratique sexuelle déviante: la nécrophilie, soit l'attirance sexuelle pour les cadavres. Dans ce roman, l'auteure utilise la forme du journal intime pour nous introduire au cœur de la psyché de son personnage. À travers le récit raconté par Lucien N, le lecteur ou la lectrice accède à ses fantasmes et à ses désirs, qui sont décrits dans les moindres détails. Dans sa structure et dans sa forme, le texte de Wittkop comporte une dimension fragmentaire et expérimentale. Un autre élément essentiel de ce roman repose sur l'aspect visuel. Le texte est en effet ponctué de collages - créés par l'auteure elle-même - qui viennent ajouter, ou du moins illustrer, le caractère inquiétant du récit. À travers cette esthétique surréaliste, on retrouve la récurrence de plusieurs images : la tête de mort, le corbeau et aussi, à quelques reprises, le visage d'Edgar Allan Poe. Dans le cadre de cet article, le texte de Wittkop sert à mettre en lumière trois éléments précis. Dans un premier temps, il s'agit de mettre en dialogue le récit de Wittkop avec deux théorisations de la perversion. La première fait appel aux idées développées par James Penney dans The World of Perversion : Psychoanalysis and the Impossible Absolute of Desire (2006). La deuxième notion qui nous intéresse est celle que Gilles Deleuze définit comme le monde sans autrui dans Logique du sens (1969). Cette discussion nous amène aussi à mettre en parallèle l'écriture de Wittkop et la question de l'abjection développée par Julia Kristeva dans Pouvoirs de l'horreur (1980). Finalement, cette réflexion sur la perversion nous porte à aborder brièvement certains aspects de la théorie queer, notamment à travers ce que l'on appelle « the anti-social strand of queer thought » (Downing et 
Gillett 89). Par exemple, un théoricien queer comme Lee Edelman demeure fasciné par la perversion en raison de son caractère improductif et de sa non-contribution au régime hétéronormatif. Le roman de Wittkop, dans sa transgression et surtout dans sa contestation des normes dominantes, accentue ce refus de contribuer à l'hégémonie reproductive et pourrait être lu à travers le prisme de la théorie queer et de son tournant « anti-social ». Ainsi, plusieurs questions guident notre réflexion : comment la nécrophilie est-elle représentée chez Wittkop ? Quelles sont les stratégies discursives utilisées ? Comment le roman permet-il de mettre en lumière ces différentes conceptualisations de la perversion ? Comment le texte de Wittkop s'inscrit-il dans cette logique non reproductive décrite par Edelman dans son ouvrage No Future : Queer Theory and the Death Drive (2004)?

\section{Première théorisation : la déviation et l'excès du désir}

Penney affirme que le terme de perversion dérive du latin perversio. Puis, au contact de discours et de savoirs apparus lors de la modernité comme la psychanalyse et la sexologie, la perversion en est venue à désigner selon lui «a deviation which, notably, is logically dependent on the norm from which it deviates » (2). Ainsi, la perversion représente une déviation par rapport à une norme. Ensuite, il discute de la relation complexe qu'il y a entre la perversion et le désir. Il opère une distinction importante entre la nature perverse du désir et la structure même de la perversion. Il affirme : « more fundamentally than "sexual difference" or "the mother's castration" what the pervert disavows is the essential perversity of desire » (1). Autrement dit, le pervers ne cherche pas seulement à désavouer la différence des sexes à travers le complexe de castration ${ }^{2}$, mais aussi à épouser la perversité essentielle du désir. De plus, l'autre conclusion avancée par Penney est la suivante : «desire is infinite and impossible. Desire always exceeds its object and articulates itself transcendentally - in a manner which overflows the container of its own sociohistorical conditions of possibility »(33). Selon lui, le désir est infini et impossible et se définit toujours à travers un excès.

Après cette première définition de la perversion, construite autour de l'essence perverse du désir et de la déviation par rapport à une norme, nous souhaitons regarder de plus près certains éléments liés au fantasme. En effet, ce dernier occupe une fonction essentielle dans ce texte et constitue une stratégie discursive pour illustrer les mécanismes pervers à l'œuvre dans l'écriture. 
C'est au tout début du récit que le personnage de Lucien effectue la généalogie de ses fantasmes morbides :

Je ne puis voir une jolie femme ou un homme agréable sans immédiatement souhaiter qu'ils fussent morts. Jadis, dans les jours d'adolescence, je le souhaitais même avec passion, avec fureur. Il s'agissait d'une voisine, de trois ou quatre ans plus âgée que moi, une grande fille brune aux yeux verts, que j'apercevais presque tous les jours. Bien que la désirant, jamais l'idée ne me serait venue de seulement toucher sa main. J'attendais, je voulais sa mort et cette mort devenait pour moi le pôle autour duquel gravitaient toutes mes pensées. (31)

Cet extrait comporte plusieurs éléments essentiels pour notre réflexion. D’abord, le personnage entretient une relation obsessionnelle avec la mort qui vient hanter ses pensées et provoquer son désir. L'usage du verbe « gravitaient » accentue l'idée que la mort devient un pôle d'attraction qui stimule son imaginaire et son désir. Ceci nous amène à préciser le statut du fantasme dans le cadre de la perversion et à établir une distinction fondamentale : le fantasme pervers n'équivaut pas toujours à la perversion. La plupart des névrosés ont ce type de fantasmes pervers, mais ne passeront jamais à l'acte, contrairement au pervers nécrophile représenté ici par Lucien. Cependant, pour Erich Fromm, la nécrophilie constitue une perversion exemplaire puisque «c'est la vraie réalisation du fantasme, le passage du fantasme dans le réel. Le névrotique peut jouir imaginairement du fantasme, mais il se tient bien à distance de sa réalisation » (14). Un autre passage du roman permet d'aborder de manière directe les caractéristiques principales de la perversion nécrophile :

Cette nuit, j'avais poussé le fauteuil de ma chambre jusqu'en face du grand miroir vénitien que j'aime tant. J'avais placé Jérôme sur mes genoux, je mordillais sa nuque aux reflets argentés, juste entre les épaules [...]. Dans les fougères grises du miroir, parmi le givre de ses rinceaux, je voyais Jérôme danser comme une grande marionnette sous les coups de mon désir. (62)

À la lumière de cet extrait, nous constatons que le partenaire inanimé de Lucien, qui porte le nom de Jérôme, est réduit à un simple objet que l'on manipule comme s'il s'agissait d'une marionnette. Son cadavre figure ici comme un simple objet inanimé et mis en image par cette métaphore. Ce trait demeure essentiel pour manifester le désir sexuel du personnage car, dans la nécrophilie, «le désir pervers se supporte de l'idéal d'un objet inanimé » (Marone 134). De plus, comme l'affirme Fulvio Marone, la nécrophilie se caractérise «par la motivation de la possession d'un partenaire qui ne peut résister ni réagir »(126). Cependant, Fromm discute des caractéristiques de la perversion nécrophile en affirmant qu'il s'agit d'un attrait passionné pour «ce qui est mort, 
putréfié, en décomposition, morbide ; la passion de transformer ce qui est vivant en quelque chose qui est privé de vie; de détruire; de s'intéresser exclusivement à tout ce qui est purement mécanique. C'est la passion de mettre en morceaux les structures vivantes » (127). L'extrait précédent montre cet intérêt exclusif chez le pervers nécrophile pour ce qui est purement mécanique et inanimé, et le texte de Wittkop met de l'avant de manière obsessionnelle cette dimension. Ensuite, Aaron Schuster dans son essai The Trouble with Pleasure : Deleuze and Psychoanalysis (2016), propose, quant à lui, une définition de la perversion associée surtout à sa dimension théâtrale. Il affirme notamment : «the pervert (typically male) stands for clarity of consciousness and consumate self-control ; he arranges his pleasures like a theatrical performance, with himself in the role of actor, director, and spectator » (85). Ici, le sujet pervers est comparé à un metteur en scène qui joue simultanément tous les rôles, qu'il s'agisse de l'acteur, du metteur en scène ou du spectateur. Lucien occupe toutes ces fonctions puisqu'il se met lui-même en scène devant un miroir avec son «partenaire » Jérôme et qu'il joue successivement tous ces rôles. Pour approfondir certains éléments du texte et ainsi dégager trois caractéristiques de la structure perverse, la théorie de la perversion avancée par Deleuze nous paraît essentielle.

\section{Deuxième théorisation : le monde sans autrui}

Dans son texte «Michel Tournier et le monde sans autrui », qui apparait dans l'appendice de son ouvrage Logique du sens (1969), Deleuze complexifie davantage le concept de perversion. Pour ce faire, il prend appui sur un texte littéraire, celui de Tournier, Vendredi ou les limbes du Pacifique, pour élaborer sa théorie. Selon le philosophe français, le roman représente « une déviation fantastique de notre monde, sous l'influence d'une sexualité transformée » (276). Il dit ensuite que le récit a quelque chose de déviant : «Robinson ne fait rien de pervers à proprement parler; et pourtant comment se débarrasser de l'impression qu'il est pervers lui-même, c'est-àdire suivant la définition de Freud, celui qui dévie par rapport aux buts ? (276) Cette réflexion l'amène par la suite à aborder directement la perversion. Pour lui, la perversion représente un concept «bâtard, mi-juridique, mi-médical » (277). Elle entretiendrait aussi, selon lui, un rapport très ambigu avec le domaine de la justice et de la médecine : «il semble qu'on cherche dans une structure de la perversion même la raison de son rapport éventuel très ambigu, tant avec la justice qu'avec la médecine » (353). Or, le personnage de Lucien se confronte à quelques reprises à l'éventualité de faire face à la justice, surtout lorsqu'il affirme : «j'ai souvent l'impression qu'on 
m'observe, qu'on me guette. Surtout les personnes de service, femmes de ménage, concierges, commerçants du quartier. Et les flics, bien sûr. Surtout les flics » (49). Lucien, dans cet extrait, se sent donc constamment surveillé et épié, mais cela ne l'empêche tout de même pas de continuer à commettre les actes dont il se réclame. En dépit de cette ambiguïté entre le juridique et le médical, c'est véritablement en fonction du désir que Deleuze définit la perversion :

La perversion ne se définit pas par la force d'un désir dans le système des pulsions ; le pervers n'est pas quelqu'un qui désire, mais qui introduit le désir dans un tout autre système et lui fait jouer, dans ce système, le rôle d'une limite intérieure, d'un foyer virtuel ou d'un point zéro (la fameuse apathie sadique). Le pervers n'est pas plus un moi qui désire, que l'Autre, pour lui, n'est un objet désiré doué d'existence réelle. (277)

Le philosophe, dans cette définition, montre que le sujet pervers introduit le désir dans une tout autre logique, qui diffère de celle associée à la névrose ou à la psychose. Deleuze va ensuite beaucoup plus loin dans sa démonstration lorsqu'il situe le pervers dans un monde sans autrui. Il dit en effet que le pervers évolue dans un monde où autrui n'est pas considéré et où la structure «Autrui » est manquante, voire absente. Selon l'auteur, Autrui «n'est ni un objet dans le champ de ma perception ni un sujet qui me perçoit : c'est d'abord une structure du champ perceptif, sans laquelle ce champ dans son ensemble ne fonctionnerait pas comme il le fait » (281). Autrui, pour Deleuze, ne représente pas un objet ou un sujet, mais il s'agit plutôt d'une structure perceptive car, comme le note Schuster, «the Other, - which Deleuze defines not as a concrete person or other ego but, in phenomenological terms, as an a priori structure which organizes the perceptual world and assures the identities of subjects and objects that appear within it - is replaced by an elemental sexuality composed of libidinal flows and partial objects » (88). Ainsi, toujours selon Deleuze, on doit chercher Autrui par ses effets : «autour de chaque objet que je perçois ou de chaque idée que je pense, l'organisation d'un monde marginal, d'un manchon, d'un fond, où d'autres objets, d'autres idées peuvent sortir suivant des lois de transition qui règlent le passage des uns aux autres » (278). Ce premier effet trouve aussi sa concrétisation lorsque l'on réfléchit à propos du désir. Selon le philosophe, c’est toujours par autrui que passe notre désir, car «je ne désire rien qui ne soit vu, pensé, possédé par un autrui possible. C'est là le fondement de mon désir. C'est toujours autrui qui rabat mon désir sur l'objet » (280).

Une autre caractéristique du pervers est ici énoncée par Deleuze : «c'est parce qu'il manque de la structure Autrui, et vit sous une tout autre structure servant de condition à son monde vivant, qu'il appréhende les autres soit comme des victimes soit comme des complices, mais dans 
aucun des deux cas ne les appréhende comme des autruis » (300). En effet, à plusieurs reprises dans le texte, Lucien appréhende les autres comme de simples victimes dont il peut abuser et jouir à sa guise. À un moment précis du roman, Lucien décide d'aller à Naples où il visite les Catacombes de San Gaudisio. Il se produit alors quelque chose d'inusité : il surprend une fidèle en train d'embrasser un crâne et il va désirer, pour la toute première fois, une femme vivante. Ceci l'amène par la suite à passer à l'acte sans son consentement :

Comme je m'engageais dans une galerie moins fréquentée, mon attention fut soudain sollicitée par le manège d'une des fidèles. [...] Un genou posé sur la chaise au dossier de laquelle elle s'accoudait, la croupe saillante, le cou tendu elle approchait son visage jusqu'à toucher une tête de mort posée sur la cimaise. [...] La femme avait réussi à introduire dans la mâchoire sa langue que je voyais à contre-jour, lécher et frétiller entre les dents du mort. [...] Ce que le spectacle et le lieu avaient d'insolite, joint à l'euphorie ressentie dès mon entrée dans les catacombes, me causèrent l'effet auquel un nécrophile peut s'attendre. Je désirai cette femme, bien qu'elle fût vivante. Je relevai sa robe noire et, écartant une culotte de coton, je découvris un large fessier poli et diaphane comme la cire des cierges environnants. Il était encore plus lisse au toucher qu'à la vue. Ayant glissé la main dans sa fente, j'en retirai mes doigts mouillés d'une liqueur opaline qui me déconcerta. (81)

Lucien surprend ici une fidèle commettre un acte pervers et ceci attise son désir. Cela l'amène même à vouloir la violer. Il n'opère plus de distinction entre les corps inanimés et amorphes qu'il viole sans remords et le corps vivant de la fidèle. L'écriture de Wittkop acquiert ici une dimension pornographique à travers la description poétique de l'acte commis par Lucien. Par exemple, son fessier est comparé à quelque chose de poli et de diaphane comme la cire des cierges. Néanmoins, c'est véritablement sa réaction à la suite de son geste qui nous permet d'affirmer que, pour lui, les autres sont considérés comme de simples victimes. Lucien évolue donc dans un monde où autrui a complètement disparu :

La femme se retourna vers moi, le visage distordu de colère :

- Je vais dire que vous avez essayé de me faire violence.

J'ignore pourquoi le dépit la portait à me menacer ainsi. En tout cas, je m'éloignai aussi vite que je le pus. (81)

Lucien ne comprend absolument pas la réaction colérique de la femme car, pour lui, il s'agit d'un acte décent et il choisit ensuite de s'éloigner rapidement. Dans ce contexte, la fidèle est littéralement désubjectivisée aux yeux de Lucien. En effet, elle acquiert le statut de simple objet qu'il peut utiliser et manipuler à sa guise. 
Deleuze résume sa théorie de la perversion en prenant comme référence les idées de Jacques Lacan. Le psychanalyste cherche pour sa part à comprendre la nécessité des comportements pervers à partir d'une structure psychique :

Le désir subit une sorte de déplacement dans cette structure, et la cause du désir se détache ainsi de l'objet; la différence des sexes est désavouée par le pervers, au profit d'un monde androgyne des doubles; sur l'annulation d'autrui dans la perversion, sur la position d'un «au-delà de l'Autre » ou d'un Autre qu'autrui, comme si autrui dégageait aux yeux du pervers sa propre métaphore; sur la « désubjectivation » perverse - car il est certain que ni la victime ni le complice ne fonctionnent comme des autruis. (299-300)

À partir de cette citation, nous dégageons ici trois caractéristiques associées à la perversion. La première stipule qu'il y a dans la structure perverse une rupture entre la cause du désir et son objet. La deuxième caractéristique repose sur la différence sexuelle qui est désavouée au profit d'un monde ambigu où règne le double. Finalement, comme nous l'avons vu dans le passage précédent, il y a une totale annulation d'autrui dans la perversion. Deux extraits du texte nous permettent d'insister sur la deuxième caractéristique dégagée par Deleuze. Les notions de paradoxe, d'ambiguïté et de brouillage apparaissent ici clairement dans le texte de Wittkop et nous mettent progressivement sur la piste d'une lecture queer. Par exemple, le texte de Wittkop aborde cet aspect ambivalent lorsqu'un client entre dans la boutique de Lucien et tente de le séduire :

Hier, un de mes clients, jeune et charmant pianiste, a tenté de me séduire [...].

- Oh... Lucien. Vous n'aimez donc pas les garçons ? Moi qui croyais...

- Mais si, bien sûr, j'aime les garçons. Et même aussi les femmes.

Ne pouvant vraiment pas lui dire : «J'aimerais beaucoup vos yeux révulsés, vos lèvres muettes, votre sexe glacial, si seulement vous étiez mort. Malheureusement, vous avez le très mauvais goût d'être en vie ». (51)

On remarque dans ce court dialogue que le personnage de Lucien ne possède pas vraiment d'intérêt envers ce jeune client qui essaye de le séduire. De plus, on constate que pour lui, la différence sexuelle importe peu : son désir est plutôt orienté vers un simple objet représenté par la figure du cadavre tout au long du texte. Lucien choisit ses partenaires sans égard à leur sexe et à leur âge. Ainsi, le cadavre agit véritablement comme un objet inanimé qui vient remplacer le but sexuel normal. Dans ce contexte, il fonctionne presque à titre de fétiche ${ }^{3}$. Dans ce passage, Lucien préfère plutôt fantasmer sur la mort de son client et sur son éventuelle inertie. Un deuxième extrait nous permet d'aborder de manière plus précise cet aspect de l'androgynie et du double lorsque Lucien découvre les cadavres de jumeaux morts noyés : «lorsque j’eus allongé les adolescents suédois sur mon lit, je ne regrettai pas ma peine. Ils devaient avoir seize ou dix-sept ans et je n'ai jamais 
rien vu d'aussi beau que ces deux-là. Ils se ressemblaient d'indicible façon et sans doute étaientils jumeaux » (87). Notre attention se porte ici sur le terme indicible qui accentue, selon nous, l'indiscernabilité du corps des jumeaux. Puis, la description physique des deux jumeaux se poursuit : «tous deux avaient de longs corps asexués - la virilité du garçon à peine profilée, les seins de la fille quasi inexistants mais infiniment désirables et qui évoquaient à mes yeux je ne sais quelle angélique nature » (87). En effet, il devient impossible pour Lucien de déterminer et de distinguer le sexe des jumeaux, car ils possèdent tous les deux des corps asexués. Dans cette perspective, nous voyons que pour le personnage, le sexe de ses partenaires lui importe peu et, dans ce contexte, la différence sexuelle est complètement déniée. Nous rappelons que la dénégation constitue le processus psychique à l'œuvre dans la perversion, contrairement au refoulement dans la névrose ou à la forclusion dans la psychose. Il s'agit selon Freud «d'un mécanisme psychique par lequel le tout petit enfant se protège de la menace de castration; il répudie alors, il désavoue, il dénie donc l'absence de pénis chez la fille, la femme, la mère » (Chemama 69).

Pour conclure cette partie, revenons à Deleuze, qui résume sa pensée sur la perversion et le monde sans autrui comme suit :

Le monde du pervers est un monde sans autrui, donc un monde sans possible. Autrui, c'est ce qui possibilise. Le monde pervers est un monde où la catégorie du nécessaire a complètement remplacé celle du possible : étrange spinozisme où l'oxygène manque [...]. Toute perversion est un autruicide, un altrucide, donc un meurtre des possibles. Mais, l'altrucide n'est pas commis par le comportement pervers, il est supposé dans la structure perverse. (301)

Comme nous l'avons vu, le monde sans autrui pour Deleuze en est un où la catégorie du nécessaire s'est écroulée et a été remplacée par celle du possible. Lucien s'exprime à un moment précis du texte sur cet autruicide lorsqu'il affirme : « car, si les nécrophiles ils sont si rares, se reconnaissent, ils ne se recherchent pas. Ils ont définitivement choisi l'incommunicabilité et leurs amours transcendent dans l'inexprimable. Il n'y a pas de lien. Solitaires, nous ne sommes même pas le lien entre la vie et la mort. Car la vie et la mort sont unies à jamais, indissociables comme l'eau mélangée au vin » (23-24). Lucien nous décrit ici le système dans lequel il évolue et qui repose sur l'incommunicabilité. Ainsi, les actes de Lucien sont commis en solitaire, en totale rupture avec l'ordre social, à l'instar du personnage de Robinson Crusoé, abandonné sur son île tel que décrit par Tournier dans son roman Vendredi ou les limbes du Pacifique. En somme, à travers la théorisation du monde sans autrui par Deleuze, il a été possible de dégager trois caractéristiques 
de la perversion et le roman nous a permis d'éclaircir ces aspects. Ceci nous amène à aborder les deux derniers aspects de notre étude : l'abjection et le tournant antirelationnel proposé par le théoricien queer Edelman.

\section{L'abject et la perversion}

Kristeva affirme que l'abjection ne se situe pas uniquement dans une absence de propreté ou de santé mais, au contraire, elle concerne « ce qui perturbe une identité, un système, un ordre. Ce qui ne respecte pas les limites, les places, les règles. L'entre-deux, l'ambigu, le mixte » (12). Selon Kristeva, l'abject est donc lié à la perturbation de l'identité ou d'un système. De plus, le cadavre et le déchet sont pour Kristeva l'exemple suprême de l'abjection : «tel un vrai théâtre, sans fard et sans masque, le déchet comme le cadavre m'indiquent ce que j'écarte en permanence pour vivre. [...] J'y suis aux limites de ma condition de vivant. De ces limites se dégage mon corps comme vivant »(11). Wittkop, avec ce roman, confronte le lecteur à ses limites. Selon Kristeva, l'abjection entretient aussi des liens étroits avec la jouissance. Celle-ci consiste à faire « exister l'abject comme tel. On ne le connaît pas, on ne le désire pas, on en jouit, violemment et avec douleur » (17). L'abjection est également une notion ambiguë, associée à l'affect, qui interroge nos limites corporelles et subjectives. L'abject est donc quelque chose qui inquiète, fascine et résiste. Par exemple, le passage suivant illustre cette dimension de l'abjection à l'œuvre dans l'écriture de Wittkop: «Tandis que je me glissais dans cette chair si froide, si douce, si délicieusement étroite qu'on ne trouve que chez les morts, l'enfant a brusquement ouvert un œil, translucide comme celui d'une pieuvre et, dans un épouvantable borborygme, elle a rejeté sur moi le flot noir d'un mystérieux liquide » (10). L'abjection dans cet extrait repose principalement sur deux aspects. Le premier concerne la relation du personnage de Lucien avec sa victime, qui est ici le cadavre d'une enfant. Le deuxième repose sur la présence de fluides qui émanent du corps mort de l'enfant et qui renvoient bien sûr à des déjections. Le roman de Wittkop évoque à plusieurs reprises la décomposition des corps morts, ce qui contribue à accentuer la dimension abjecte de l'écriture. L'extrait suivant, dans lequel le personnage de Lucien fait prendre le bain à sa victime, le montre très bien : «ses chairs s'amollissent d'heure en heure, son ventre verdit, s'effondre, grouille de flatulences mauvaises qui crèvent en énormes bulles dans l'eau du bain » (33).

Dans le sillage de notre discussion sur la perversion, cette courte réflexion sur l'abjection nous amène à discuter brièvement de la présence de ces questions à travers des discours 
contemporains. Pour ce faire, nous ferons appel notamment à la théorie queer. Rappelons que le mot queer provient d'une insulte qui désigne le déviant, le pervers et tout ce qui déroge des conduites sexuelles normales ou hétéronormatives. Dans son texte, «Le mouvement "queer" : des sexualités mutantes ? », Pascale Macary revient sur le terme qui, selon elle, se définit comme une façon de «nommer l'autre dans son étrangeté, sa bizarrerie. Étymologiquement, ce signifiant renvoie à un "travers", qui s'oppose dans la langue anglaise moderne à straight (droit, "hétérosexuel" dans le champ de la sexualité)»(3). Aujourd'hui, le terme queer est surtout employé pour désigner «toute pratique transgressant les classifications en vigueur, les représentations traditionnelles, les normes sexuelles » (3). Comme nous l'avons vu, le récit de Lucien entre en résonance avec cette définition du queer puisque les actes qu'il commet ébranlent et déstabilisent les normes traditionnelles de la sexualité.

\section{La pulsion de mort et la logique non reproductive}

Dans son ouvrage No Future: Queer Theory and the Death Drive, Edelman discute, à l'aide d'une perspective psychanalytique lacanienne, de la pulsion de mort pour réfléchir sur ce qui caractérise le queer. L'auteur prend des exemples empruntés à la littérature et au cinéma afin d'analyser la manière dont la figure de l'enfant devient une métaphore pour exprimer un futur reproductif (reproductive futurism). Le queer, pour sa part, s'associe à la négativité, notamment dans ce souci de mettre en péril l'ordre Symbolique ${ }^{4}$, car, comme il l'affirme : «the efficacy of queerness, its real strategic value, lies in its resistance to a Symbolic reality that only ever invests us as subjects » (18). Dès l'introduction, il avance : «queerness names the side of "those not fighting for the children," the side outside the consensus by which all politics confirms the absolute value of reproductive futurism » (3). Autrement dit, l'enfant, pour Edelman, est toujours associé à une logique reproductive symbolisant l'espoir et l'innocence pour la société hétéronormative. Dans ce contexte, la figure positive de l'enfant doit absolument être protégée des déviations du monde extérieur. Le queer s'oppose à cette logique reproductive et constitue plutôt une forme de résistance. Dans le texte de Wittkop, Lucien échappe bien sûr à cette logique reproductive puisqu'il a des relations sexuelles, d'une part, avec des cadavres et, d'autre part, avec quelques enfants. Il met donc en péril les fondements de la société à travers les actes qu'il commet. L'innocence et la pureté, habituellement associées à l'enfant, sont ici détournées, voire anéanties, à travers des actes sexuels d'une grande indécence comme en témoigne l'extrait suivant : «il a un vrai corps pour 
jouer avec, pour jouir avec, encore que jeux et jouissances doivent se dérouler sur les surfaces externes. Cet enfant est si étroit qu'il m'a fallu renoncer à des délices plus profonds » (33). Edelman introduit par ailleurs la pulsion de mort qui, selon lui, s'associe également au projet queer : « the death drives names what the queer, in the order of the social, is called forth to figure : the negativity opposed to every form of social viability » (9). Cette citation d'Edelman nous parait ici intéressante étant donné que la pulsion de mort désigne une négativité qui s'oppose à toute forme de sociabilité. Un peu plus tôt dans le roman, Lucien évoquait déjà les rapports ambigus du désir et de la mort lorsqu'il faisait son entrée dans le cimetière Montparnasse : «l'immense foule endeuillée se pressait dans les allées, parmi des gloires de chrysanthèmes et l'air avait la saveur amère, enivrante de l'amour. Éros et Thanatos. Tous ces sexes sous la terre, y pense-t-on jamais ? » (15). Wittkop fait directement référence aux deux pulsions évoquées par la psychanalyse et reprises par Edelman dans son argumentaire, soit Éros, la pulsion de vie, et Thanatos, la pulsion de mort. De plus, le queer selon Edelman, à travers cette valorisation de la négativité et de la nonreproductivité, comporte aussi une dimension abjecte qui entre en résonance avec les théories de Kristeva évoquées précédemment : «queerness [...] figures, outside and beyond its political symptoms, the place of the social order's death drive : a place to be sure of abjection expressed in the stigma » (3). Edelman introduit par la suite le concept de jouissance dans son argumentation. Comme nous l'avons vu chez Kristeva, la jouissance constitue un mouvement qui va au-delà de la signification et qui, dans la théorie lacanienne, s'associe au Réel ${ }^{5}$. Il affirme à ce propos :

Queerness, therefore, is never a matter of being or becoming but, rather, of embodying the remainder of the Real internal to the Symbolic order. One name for this unnameable remainder, as Lacan describes it, is jouissance, sometimes translated as enjoyment : a movement beyond the pleasure principle, beyond the distinctions of pleasure and pain, a violent passage beyond the bounds of identity, meaning and law. (25)

Cette définition de la jouissance formulée ici par Edelman, caractérisée comme un mouvement qui dépasse la distinction entre plaisir et douleur ou les liens qui unissent l'identité, la loi et la signification, fait écho à la pratique nécrophile perpétrée par Lucien tout au long du texte. La jouissance qui constitue un au-delà du plaisir est éminemment queer pour Edelman puisqu'elle est à la fois indéfinissable et qu'elle résiste aux discours normalisateurs.

Pour conclure, le texte de Wittkop, Le Nécrophile, nous a fourni l'occasion de discuter des différentes théories de la perversion de Penney et de Deleuze. Nous remarquons ici qu'un texte littéraire peut contribuer à enrichir, à nourrir et à illustrer un phénomène complexe comme la 
perversion. Cependant, la théorie queer, avec son tournant antirelationnel, nous a permis de dégager le potentiel déstabilisant du roman de Wittkop et son refus évident de correspondre aux normes sociales. Nous constatons en effet une similitude entre les différentes théorisations de la perversion que nous avons soulevées et le tournant antisocial de la théorie queer. Par exemple, la perversion possède une dimension antiproductive qui se rapproche de ce qu'Edelman théorise. Ensuite, avec l'apport de Kristeva, nous avons montré que l'abjection venait confronter le lecteur à ses propres limites. La nécrophilie, cette attirance sexuelle pour la mort et l'inanimé, constitue une posture radicale, voire anarchique, qui vient transgresser toutes les règles de la société. Comme nous le rappelle Macary, l'approche queer permet notamment d'étudier un texte littéraire dans le but de dépasser « la simple étude de l'homosexualité dans la littérature : il débusque ce qui est "pervers" dans les textes, il étudie les stratégies par lesquelles les œuvres subvertissent les

catégorisations sexuelles et le système de genre » (5). Or, le texte de Wittkop participe à cette subversion des normes en matière de sexualité puisqu'il aborde de manière directe une pratique sexuelle inusitée qui rompt avec l'hétéronormativité et la reproduction sociale. Le premier roman de Wittkop contribue à subvertir les catégorisations sexuelles et à explorer les facettes les plus sombres de la psyché humaine.

\section{Bibliographie}

Chemama, Roland. Dictionnaire de la psychanalyse. Paris : Larousse, 1995.

Deleuze, Gilles. Logique du sens. Paris : Minuit, 1969.

Downing, Lisa et Gillett, Robert. «Georges Bataille at the Avant-garde of Queer Theory? Transgression, Perversion and Death drive ». Nottingham French Studies 50.3 (2011) : 89102.

Edelman, Lee. No Future : Queer Theory and the Death Drive. Londres : Duke UP, 2004.

Freud, Sigmund. La vie sexuelle. Paris : PUF 1997.

---. Trois essais sur la théorie sexuelle. Paris : Gallimard, 1905.

Fromm, Erich. The Anatomy of Human Destructiveness. New York : Holt, 1973.

Kristeva, Julia. Pouvoirs de l'horreur : essais sur l'abjection. Paris : Gallimard, 1980.

Macary-Garipuy, Pascale. «Le mouvement "queer": des sexualités mutantes?». Psychanalyse 7.3 (2006) : 43-52.

Marone, Fulvio. « L'amour d'un corps mort». L'en-je lacanien 2.17 (2011) : 121-139. 
Penney, James. The World of Perversion : Psychoanalysis and the Impossible Absolute of Desire. Albany : State U of New York P, 2006.

Schuster, Aaron. The Trouble with Pleasure : Deleuze and Psychoanalysis. Cambridge : The MIT P, 2016.

Wittkop, Gabrielle. Le Nécrophile. Paris : Verticale, 1972.

\footnotetext{
Notes

${ }^{1}$ Un seul mémoire de maîtrise analyse l'œuvre de Wittkop en relation avec deux autres auteurs. Il s'agit de celui de Julie Delorme à l'Université d'Ottawa qui porte le titre «Le tabou et ses représentations littéraires : Ma mère de Georges Bataille, Le Nécrophile de Gabrielle Wittkop et Putain de Nelly Arcan ». Il existe également un nombre restreint de publications ou d'études sur l'écrivaine.

${ }^{2}$ Rappelons que le complexe de castration désigne dans la théorie psychanalytique « l'ensemble des conséquences subjectives, principalement inconscientes, déterminées par la menace de castration chez l'homme et par l'absence de pénis chez la femme » (Chemama 41).

${ }^{3}$ Sigmund Freud aborde la perversion dans deux textes : Trois essais sur la théorie sexuelle (1905) et Le fétichisme (1927). Dans les Trois essais sur la théorie sexuelle, Freud introduit deux termes : l'objet sexuel et le but sexuel. L'objet sexuel désigne « la personne dont émane l'attraction sexuelle » (38), tandis que le but sexuel concerne « l'acte auquel pousse la pulsion » (38). À partir de ces deux paramètres, Freud constate que ces deux pôles subissent de nombreuses déviations. Le fétichisme sera abordé par Freud dans le deuxième groupe, soit celui des déviations par rapport au but sexuel. Le but sexuel se décrit comme « l'union des parties génitales dans l'acte appelé accouplement » (57). Freud affirme que le fétiche représente soit « une partie du corps qui convient en général très mal à des buts sexuels (pied, chevelure) » (62) ou « un objet inanimé dont on peut démontrer la relation avec la personne sexuelle qu'il remplace » (62). Néanmoins, pour Freud, le fétiche d'un sujet devient véritablement l'objet d'une perversion et mérite une attention clinique seulement s'il « s'installe à la place du but normal, ou encore lorsque le fétiche se détache d'une personne déterminée pour devenir l'unique objet sexuel »(63). Ensuite, dans son texte de 1927, intitulé Le fétichisme, Freud approfondit encore davantage sa réflexion sur la perversion fétichiste. Il avance notamment que le fétiche «est un substitut du pénis » (133). Selon Freud, il ne s'agit pas de n'importe quel pénis, mais il s'agit plutôt du substitut « du phallus de la femme (la mère) auquel a cru le petit enfant et auquel, nous savons pourquoi, il ne veut pas renoncer » (134).

${ }^{4}$ Le Symbolique dans la théorie lacanienne désigne une «fonction complexe et latente qui embrasse toute l'activité humaine, comportant une part consciente et une part inconsciente, qui est attachée à la fonction du langage et plus spécialement à celle du signifiant » (Chemama 323).

${ }^{5}$ Le Réel chez Lacan se définit comme « l'impossible, il est ce qui ne peut être complètement symbolisé dans la parole ou l'écriture et, par conséquent, ne cesse pas de ne pas s'écrire » (278).
} 